2. Verhalten des Chlorkalks bei nach und nach erfolgender Behandlung mit Wasser, nebst Bemerkungen in Betreff seiner Constitution.

Im festen Chlorkalke nehmen viele Chemiker ein Gemenge von unterchlorigsaurem Kalk mit Chlorcalcium und Kalkhydrat, andere eine Verbindung der beiden Salze, gemengl mit Kalkhydrat, an, während Millon den Chlorkalk als $\mathrm{Ca}\left\{\begin{array}{l}\mathrm{Cl} \\ \mathrm{Cl}\end{array}\right.$ mengt mit Kalkhydrat, glaubte betrachten zu können. - Das Verhalten des Chlorkalks beim fortgesetzten Behandeln mit kleineren Wassermengen schien geeignet, in dieser Frage einiges Licht zu geben. Ich ersuchte daher Herrn F. R o s e aus Lippstadt, in der genannten Beziehung eine Reihe von Versuchen anzustellen, und es löste derselbe die Aufgabe mit eben so viel Ausdauer als Sorgfalt und Geschick.

Ich theile im Folgenden zuerst die Art mit, wie die Untersuchung ausgeführt wurde, sodann die Resultate, welche sie lieferte, und endlich die Schlüsse, welche sich aus diesen ziehen lassen.

1) Der zur Untersuchung verwandte Chlorkalk war frisch und aus der Mitte eines 5 Centner enthaltenden Fasses genommen. Er enthielt, nach Bunsen's Methode geprüft, im Mittel dreier gut übereinstimmender Versuche $16,25 \mathrm{pC}$. unterchlorige Säure, entsprechend $26,52 \mathrm{pC}$. wirksamen Chlors nach gewöhnlicher Bezeichnungsweise. - Zur Bestimmung des gesammten Chlors wurde eine abgewogene Menge Chlorkalk zunächst mit Wasser und Ammoniak andauernd erwärmt. Nachdem so die unterchlorige Säure vollständig zerstört war, säuerte man mit Salpetersäure schwach an, stumpfte den Ueberschufs mit kohlensaurem Natron vorsichtig ab und bestimmte das Chlor unter Anwendung von chromsaurem Kali mit Zehntel-Normal-Silberiösung. 
0,247 Grm. erforderten 20,6 CC., entsprechend 29,57 pC. gesammtem Chlor. Durch Abziehen des in der unterchlorigen Säure enthaltenen Chlors von der gesammten Chlormenge liefs sich das an Calcium gebundene Chlor ermitteln. - Der Kalk wurde mit oxalsaurem Ammon gefällt. In zwei Bestimmungen erhielt man 46,35 und 46,40, im Mittel 46,37 pC.

Aus diesen Zahlen berechnet sich folgende procentische Zusammensetzung :

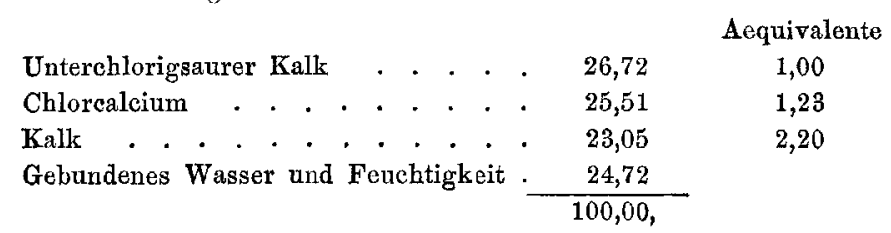

welche man zur Gewinnung einer besseren Uebersicht auch so darstellen kann :

\begin{tabular}{|c|c|c|}
\hline \multicolumn{3}{|l|}{$\begin{array}{l}\text { Unterchlorigsaurer Kalk } \cdot \dot{\cdot} \cdot \cdot \cdot \cdot \cdot \text {. } \\
\text { Chlorcalcium, zum unterchlorigsaurem Kalk }\end{array}$} \\
\hline im Verhältnifs 1 Acq. : 1 Aeq, stehend & 20,72 & 1,00 \\
\hline Chlorcalcium, überschüssiges . . . . . & 4,79 & 0,23 \\
\hline Kalkhydrat $(\mathrm{CaO}, \mathbf{H O}) \cdot$. & 30,46 & 2,20 \\
\hline Weiteres gebundenes Wasser u. Feuchtigkeit & 17,31 & \\
\hline & 100,0 & \\
\hline
\end{tabular}

Die Richtigkeit dieser Zusammenselzung wurde auf folgende Weise controlirt :

0,5352 Grim. Chlorkalk brachte man in einen Kolben, fügte 15 CC. Normal-Salzsäure hinzu und kochte gelinde, während durch ein mehrere Fufs langes, schief aufwärts gerichtetes Glasrohr das Enlweichen von Salzsäure verhindert wurde. Nachdem alles Chlor ausgetrieben war, titrirte man mit Normalnatronlauge zurück und gebrauchte $6,6 \mathrm{CC}$, somit waren $15-6,6=8,4$ Salzsäure gebunden oder zersetzt. Ein zweiter Versuch lieferte fast genau dasselbe Resultat. 100 Grm. Chlorkalk hätten somit 1569 CC. Normal-Salzsäure, entsprechend 57,206 Chlorwasserstoff, gebunden oder zerstört. 
Es entsprechen nun :

a. Die in dem Chlorkalke vorhandene, aus $26,72 \mathrm{CaO}, \mathrm{ClO}$ und 20,72 $\mathrm{CaCl}$ bestehenden $47,44 \mathrm{pC}$. normalen ätzkalkfreien Chlorkalkes wasserfreiem Chlorwasserstoff $\operatorname{dem}(\mathrm{CaO}, \mathrm{ClO}+\mathrm{CaCl})+2 \mathrm{Aeq} . \mathrm{ClH}=2 \mathrm{CaCl}$ $+2 \mathrm{HO}+2 \mathrm{Cl}$.

b. Die 23,05 Kalk (oder 30,46 Kalkhydrat) . . . .

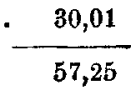

welche Zahl mit der direct gefundenen $(57,206)$ fast vollkommen gleich ist. Der den 4,79 Chlorcalcium der obigen Zusammenstellung entsprechende Kalk hatte somit keine Salzsäure in Anspruch genommen, woraus folgt, dafs das genannte Chlorcalcium als, im Hinblick auf den vorhandenen unterchlorigsauren Kalk, überschüssiges Chlorcalcium zugegen war.

2) Da es sich bei vorläufigen Versuchen herausgestellt hatte, dafs der Chlorkalk auf einem Filter sich nicht auswaschen liefs, indem sich die Poren des letzteren sehr bald verstopften, verfuhr man zur allmäligen Extraction desselben mit Wasser auf folgende Weise :

$50 \mathrm{Grm}$. wurden mit etwa $80 \mathrm{CC}$. Wasser zu einem dünnen Brei angerieben und dieser auf ein faltiges Filter gebracht. Das in der Reibschale Anhaftende entfernte man möglichst mit einem Hornspatel; Nachspülwasser wurde nicht angewandt. Bei den zwei angestellten Versuchsreihen ergaben sich ungefähr $20 \mathrm{CC}$. Filtrat. - Nach vollständigem Abtropfen wurde das Filter aus dem Trichter genommen, auf einer Glasplatte ausgebreitet und das darauf Befindliche mit dem Hornspatel und durch Absprizen in die (ausgewaschene) Reibschale gebracht. Nachdem es wieder mit Wasser angerieben war, brachte man es auf ein neues faltiges Filter. Bei beiden Versuchsreihen ergaben sich jetzt etwa 30 CC. Filtral. Nach dem Ablaufen verfuhr man wieder wie oben. Dieses dritte Filtrat betrug wenig mehr als $100 \mathrm{CC}$. Vom vierten 
Abreiben erhielt man etwa 120, vom fünften 150, vom sechsten und siebenten elwas mehr, vom achten über 200, vom neunten und (nur bei der ersten Versuchsreihe) zehnten über 300 CC. - Genaues Nachmessen der Filtrate zum Behufe einer Vergleichung der Summe ihrer Gehalte mit dem des verwandten Chlorkalks war zwecklos, da jedesmal in der Reibschale und auf den Filtern kleine Quantitäten hängen blieben und verloren gingen. Delshalb wurden auch nur annähernd gleiche Mengen Wasser zu den gleichen Filtratnummern beider Versuchsreihen verwandt.

Um Zersetzung der erhaltenen Lösungen beim Stehen an der Luft zu vermeiden, versäumte man nie, die Filtrate unmittelbar nach dem Abtropfen zu analysiren. Die unterchlorige Säure wurde bei den Filtraten 1 bis 8 inclusive mit P e n ot'scher Lösung, von der 1000 CC. 3,1776 Grm. Chlor oder 1,9473 Grm. unterchloriger Säure entsprachen, bestimmt, bei den sehr verdünnten Filtraten 9 und 10 dagegen nach der Bunsen'schen Methode, - die gesammte Menge des Chlors bestimmte man so, wie es oben bei der Analyse des Chlorkalks angegeben worden ist. Der mit Wasser erschöpfte Rückstand wurde schliefslich mit $100 \mathrm{CC}$. Wasser zu einer gleichmäfsigen Milch angerieben und auch diese in beschriebener Weise auf unterchlorige Säure und Gesammtchlor geprüft.

3) Die Resultate der so ausgeführten Analysen sind im Folgenden zusammengestellt.

Versuchsreihe I.

100 Theile der Auszüge enthalten : der unterchlorigen Säure entsprechendes

Filtrat

1.

2.

3.

4.

5. unterchlorige Säure

2,7598

3,1785

2,2487

0,9735

0,2842 wirksames Chlor $(43,46: 70,92)$

4,5043

5,1875

3,6701

1,5888

0,4639 gesammtes

ChIor

12,4464

7,4820

3,5016

1,4272

0,4219 
bei nach und nach erfolgender Behandlung mit Wasser. 321

$\begin{array}{cccc}\text { Filtrat } & \begin{array}{c}\text { der unterchlorigen } \\ \text { Säure entsprechendes } \\ \text { wirksames Chlor } \\ \text { rige Säure }\end{array} & \begin{array}{c}\text { gesammtes } \\ \text { Chlor }\end{array} \\ 6 . & 0,0973 & 0,1588 & 0,1560 \\ 7 . & 0,0366 & 0,0597 & 0,0610 \\ 8 . & 0,0078 & 0,0127 & \\ 9 . & 0,0036 & 0,0060 & 0,0073 \\ \text { 10. } & 0,0023 & 0,0038 & 0,0047 \\ \text { Aufgeschlämmter } & & & 0,0895 \\ \text { Rückstand . . } & 0,0845 & 0,1379 & \end{array}$

Versuchsreihe II.

$\begin{array}{cccc}\text { Filtrat } & \begin{array}{c}\text { unterchlo- } \\ \text { rige Säure }\end{array} & \begin{array}{c}\text { der unterchlorigen } \\ \text { Süure entsprechendes } \\ \text { wirksames Chlor }\end{array} & \begin{array}{c}\text { gesammtes } \\ \text { Chlor }\end{array} \\ \text { 1. } & 2,8426 & 4,6393 & 13,3152 \\ 2 . & \mathbf{3 , 1 5 4 1} & \mathbf{5 , 1 4 7 7} & \mathbf{7 , 5 7 0 7} \\ \text { 3. } & 2,8523 & 4,6551 & 4,5566 \\ 4 . & 1,6744 & 2,7327 & 2,5708 \\ \text { 5. } & 0,8664 & \mathbf{1 , 4 1 4 0} & 1,3208 \\ 6 . & 0,3348 & 0,5465 & 0,4964 \\ \text { 7. } & 0,1255 & 0,2049 & 0,1861 \\ 8 . & 0,0365 & 0,0596 & 0,0532 \\ \text { 9. } & 0,0107 & 0,0175 & 0,0213 \\ \text { coschlämmter } & & & \\ \text { ckstand } . & 0,0915 & 0,1494 & 0,0922\end{array}$

4) Berechnet man nun die unterchlorige Säure auf unterchlorigsauren Kalk, zieht das darin enthaltene Chlor von dem Gesammtchlor ab und berechnet aus dem Reste des Chlors die Mengen des in den Filtraten enthaltenen Chlorcalciums, so erhält man folgende Resultate :

\section{Versuchsreihe I.}

100 Theile der Auszüge enthalten :

$\begin{array}{cccc}\text { Filtrat } & \begin{array}{c}\text { unterchlorig- } \\ \text { sauren Kalk }\end{array} & \begin{array}{c}\text { Chlor- } \\ \text { calcium }\end{array} & \begin{array}{c}\text { auf } 1 \text { Aeq. CaO, } \\ \text { ClO kommen so- } \\ \text { mit Aeq. CaCl }\end{array} \\ \text { 1. } & \mathbf{4 , 5 3 7 1} & \mathbf{1 5 , 9 4 3 7} & 4,5279 \\ \text { 2. } & 5,2254 & \mathbf{7 , 6 4 5 1} & \mathbf{1 , 8 8 5 1} \\ \text { 3. } & \mathbf{3 , 6 9 6 8} & \mathbf{2 , 6 0 6 4} & 0,9084 \\ 4 . & 1,6004 & 0,9897 & 0,7968 \\ \mathbf{5 .} & 0,4672 & 0,2971 & 0,8193\end{array}$

Anual. d. Ohemie u. Pharm. CXVIII. Bd. 3. Heft. 


$\begin{array}{cccc}\text { interchlorig- } & \begin{array}{c}\text { Chlor- } \\ \text { calcium }\end{array} & \begin{array}{c}\text { auf } 1 \text { Aeq. CaO } \\ \text { ClO kommen so- } \\ \text { mit Aeq. CaCl }\end{array} \\ 6 . & \begin{array}{c}\text { untrat } \\ \text { sauren Kalk }\end{array} & 0,11900 & 0,9647 \\ 7 . & 0,0602 & 0,0487 & 1,0422 \\ 8 . & 0,0128 & - & - \\ 9 . & 0,0060 & 0,0067 & 1,4319 \\ 10 . & 0,0039 & 0,0043 & 1,4521 \\ \text { Rückstand } & 0,1389 & 0,0321 & 0,2981\end{array}$

$\begin{array}{crrr}\text { 1. } & \mathbf{4 , 6 7 3 2} & \mathbf{1 7 , 1 9 7 1} & \mathbf{4}, \mathbf{7 4 1 6} \\ \text { 2. } & \mathbf{5 , 1 8 5 3} & \mathbf{7 , 8 1 5 2} & \mathbf{1 , 9 4 2 0} \\ \text { 3. } & 4,6892 & \mathbf{3 , 4 8 6 2} & 0,9579 \\ \text { 4. } & \mathbf{2 , 7 5 2 7} & 1,8838 & 0,8818 \\ 5 . & 1,4243 & 0,9599 & 0,8684 \\ \text { 6. } & 0,5504 & 0,3489 & 0,8168 \\ \text { 7. } & 0,2064 & 0,1309 & 0,8170 \\ \text { 8. } & 0,0600 & 0,0366 & 0,7856 \\ \text { 9. } & 0,0176 & 0,0196 & 1,4355 \\ \text { Rückstand } & 0,1505 & 0,0273 & 0,2341\end{array}$

5) Aus diesen Thatsachen ergeben sich nun folgende Schlüsse :

a. Die bei dem ersten Anreiben der 50 Grm. Chlorkalk mit Wasser verwandte, etwa $80 \mathrm{CC}$. Wasser betragende Wassermenge genügte vollkommen, um alles vorhandene Chlorcalcium (12,75 Grm.) zu lösen. Die ablaufenden $20 \mathrm{CC}$. Filtrat enthielten davon 3,2 Grm., die mechanisch zurückgehaltene Chlorcalciumlösung (elwa 60 CC.) enthielt den Rest, somit 9,55 Grm. - Beim zweiten Anreiben wurden etwa $65 \mathrm{CC}$. Wasser zugesetzt; es entstand somil eine Flüssigkeit, welche in elwa $125 \mathrm{CC}$ 9,55 Chlorcalcium, also ungefähr einen Procentgehalt enthielt, wie ihn das Filtrat Nr. 2 wirklich zeigte. In derselben Weise erklärt sich nun auch der im Verhältnils des weiter hinzugekommenen Wassers stets abnehmende Chlorcalciumgehalt der folgenden Filtrate.

$b$. Anders verhielt sich die Sache bei dem unterchlorigsauren Kalk. Dieser kam durch die beim ersten Abreiben angewandte Wassermenge offenbar nur unvollständig in Lö- 
sung. Das Wasser war vom Chlorcalcium schon zu sehr in Anspruch genommen, auch genügte wohl dessen Menge überhaupt nicht (die Löslichkeit reinen unterchlorigsauren Kalks ist nicht bekannt). - Beim zweiten Abreiben trat das Chlorcalcium weniger hemmend auf, und da es an ungelöstem unterchlorigsaurem Kalk nicht fehlte, so mufste das Filtrat, wie diefs auch der Fall war, reicher an unterchlorigsaurem Kalk sein, als das erste. - Beim dritten Anreiben traf das Wasser noch immer auf ungelösten unterchlorigsauren Kalk, wie der noch hohe Gehalt des Filtrates Nr. 3 erweist, aber der unterchlorigsaure Kalk reichte zur Sättigung des Wassers nicht mehr hin, wie sich daraus ersehen läfst, dafs das Filtrat Nr. 3 ärmer war als das Filtrat Nr. 2. - Da von Nro. 3 an aller unterchlorigsaure Kalk gelöst war, so nehmen nunmehr die Gehalte daran bei den weiteren Filtraten rasch ab.

c. Von der Abreibung Nr. 3 an war somit sowohl alles Chlorcalcium wie aller unterchlorigsaure Kalk gelöst, daher mufste auch das Verhältnifs zwischen Chlorcalcium und unterchlorigsaurem Kalk von da an sich gleich bleiben, was auch, von kleinen Ábweichungen abgesehen, der Fall war.

$d$. Da sich nun aus den Gehalten der Filtrate, wie gezeigt worden, ergiebt, dals das Chlorcalcium schon bei der ersten, der unterchlorigsaure Kalk aber erst bei der dritten Abreibung vollständig in Lösung kam, so ist man gezwungen anzunehmen, entweder dafs beide nur gemengt sind, oder aber, dafs sie eine durch Wasser sofort in Chlorcalcium und unterchlorigsauren Kalk zersetzbaze Verbindung bilden.

e. Was das im Chlorkalk enthaltene Kalkhydrat betrift, so halte ich dafür, dafs es mit dem Chlorcalcium zu basischem Chlorcalcium verbunden ist. Nur bei dieser Annahme erklärt es sich, wefshalb 4 Aeq. festes Kalkhydrat nur 2 Aeq. Chlor aufnehmen. Bei Einwirkung von Wasser zerfällt diese Verbindung, wie wir diefs auch an der krystallisirten Verbindung 
$3 \mathrm{CaO}, \mathrm{CaCl}+16$ aq. sehen, in sich lösendes Chlorcalcium und in Kalkhydrat.

$f$. Das ganze Verhalten des festen Chlorkalks erklärt sich somit aus der Annahme, dafs derselbe ein Gemenge sei von $1 \mathrm{Aeq}$. $\mathrm{CaO}, \mathrm{ClO}$ mit 1 Aeq. basischem Chlorcalcium von der Formel $\mathrm{CaCl}, 2 \mathrm{CaO}+4$ aq.

g. Das bei Wassereinwirkung aus dem basischen Chlorcalcium ausgeschiedene Kalkhydrat übt übrigens unverkennbar noch eine gewisse Kraft der Anziehung auf das Chlorcalcium wie auf den unterchlorigsauren Kalk aus. Dieselbe blieb sich bei den Abreibungen 3 bis 8 ziemlich gleich, von da an aber (also bei der Einwirkung gröfserer Wassermengen) liefs sie für das Chlorcalcium nach, während sie für den unterchlorigsauren Kalk fortbesland. So erklärt es sich, welshalb bei Nr. 9 und 10 auf 1 Aeq. unterchlorigsauren Kalk plötzlich 1,4 Aeq. Chlorcalcium auftreten, und wefshalb im Rückstande der unterchlorigsaure Kalk zum Chlorcalcium in ganz anderem Verhältnisse auftrat, als im letzten Filtrate, nämlich in dem $1 \mathrm{Aeq.} \mathrm{zu} 0,26 \mathrm{Aeq}$.

h. Der zuletzt erwähnte Umstand erklärt es auch, warum man bei Chlorkalkprüfungen nur dann ein richliges Resultat erhält, wenn man die durch Abreiben und Schülteln mit Wasser dargestellte gleichmäfsige Milch verwendet, während es etwas zu niedrig ausfällt, wenn man sich der durch $\mathrm{Ab}$ sitzen geklärten Lösung bedient, und etwas zu hoch, wenn man den abgeselzten dickeren Theil der Milch in Gebrauch nimmt.

R. Fresenius. 